\title{
Editorial
}

\section{Acknowledgement to Reviewers of Entropy in 2013}

Entropy Editorial Office, MDPI AG, Klybeckstrasse 64, CH-4057 Basel, Switzerland

Published: 24 February 2014

The editors of Entropy would like to express their sincere gratitude to the following reviewers for assessing manuscripts in 2013:

Abaimov, S.G.

Abass, Khaled

Abdallah, Samer

Abdul Hakim, Saidur

Abdullah, Saiful Arif

Abe, Sumiyoshi

Abel, David

Abel, Markus

Aghagolzadeh, Mehdi

Ahammer, $\mathrm{H}$.

Ahmadi, Pouria

Ahn, Choon Ki

Aitken, Jonathan

Albeanu, Grigore

Aleroud, Ahmed

Ali Hajji, Mohamed

Ali, Djafari

Ali, Giuseppe

Ali, Mazhar

Alic, Fikret

Al-Qasimi, Asma

Althunibat, Saud

Alvarez, A.

Alves Monteiro, Luiz Henrique

Alves-Kotzev, Natasha

Amari, Shun-Ichi
Amatya, R.

Amigó, José M.

Amirabdollahian, Mahsa

Anand, S.

Anastasiadis, Anastasios

Andersen, Neil G.

Andersson, Erika

Ang, Kai Keng

Angulo Brown, F.

Annila, Arto

Antoine, J.-P.

Apreutesei, Narcisa

Araújo, Nuno A. M.

Aricò, Costanza

Arima, $\mathrm{T}$.

Arnaboldi, Valerio

Arnold, Axel

Arroyo, David

Arsanjani, Jamal Jokar

Aschwanden, Markus J.

Assad, M. El-Haj

Aste, Tomaso

Athenes, Manuel

Attar, Peter J.

Attard, Phil

Atzberger, Paul
Auer, Stefan

Ausloos, Marcel

Avanak, M. R. N.

Averbeck, Bruno

Babkovskaia, N.

Badía-Majós, A

Badruddoza, Anm

Bae, Sejong

Baeyens, Enrique

Bajic, Dragana

Balabdaoui, Fadoua

Balasis, Georgios

Balduzzi, David

Baleanu, Dumitru

Balestrassi, Pedro Paulo

Balibrea, Francisco

Ballesteros, Ballesteros

Bamba, Kazuharu

Banigan, Edward J.

Baptista, M.S.

Barbera, Elvira

Barbu, Adrian

Bardera, Anton

Barreto, Ernest

Basirat Tabrizi, Hassan

Bastolla, Ugo 
Batelaan, Herman

Battaglia, Demian

Beal, Jacob

Beckwith, Andrew W.

Behnam, Hamid

Bekenstein, Jacob

Bekiros, Stelios

Benedetto, Francesco

Bennewitz, Jürgen

Beretta, Gian Paolo

Berezovcki, Arkadi

Berg, Jordan M.

Berger, Jorge

Berry, R. Stephen

Bezuijen, Adam

Bianco, Vincenzo

Bianconi, Ginestra

Bilancini, Ennio

Blasco, Andrea

Boccaletti, Stefano

boguñá, marián

Bokes, Peter

Bonakdari, $\mathrm{H}$.

Bonassi, Fernando

Boncinelli, Leonardo

Bonsignorio, Fabio

Borge-Holthoefer, Javier

Borges, E.

Borgis, Daniel

Boškoski, Pavle

Botteron, Cyril

Bouchaud, Jean-Philippe

Bountis, Tassos

Bousso, Raphael

Bowler, Michael G.

Bowman, Gregory

Bradley, Seamus

Brandt, Luca

Brask, Jonathan Bohr

Brasselet, Romain

Bratko, Dusan

Braun, Dieter
Brender, Pierre

Bresme, Fernando

Briefer, Elodie

Briers, Mark

Brodka, Piotr

Brown, Lowell

Brüggemann, Rainer

Bruin, Henk

Brunsell, N.A.

Bucki, Robert

Buono, Camila

Buonomo, Antonio

Burel, G

Burheim, Odne S.

Burioni, Raffaella

Buscema, Massimo

Bussi, Giovanni

Butler, Leo T.

Cabello, Adán

Cafagna, Donato

Cafaro, Carlo

Cai, Weihua

Cai, YanPeng

Caldas, Ibere

Callegari, Sergio

Calmet, Xavier

Camastra, Francesco

Cameron, Maria

Campa, Alessandro

Campbella, Colin

Campos, Diógenes

Cancho, Ramon Ferrer i

Cannon, William R.

Cano, Jose Ramón

Canovas Pena, Jose S.

Cao, F.J.

Carvalho, Alexandre R.F.

Caticha, Nestor

Centola, Damon

Cerbus, Rory

Chakraborti, Anirban

Chakraborty, Uday Kumar
Chandra, Rohitash

Chandrashekar, C.M.

Chaplin, Martin

Charitidis, Costas A.

Chau, Kwok-wing

Chaudhuri, Debasish

Chechkin, Aleksei

Chen, Badong

Chen, Chun-Chi

Chen, Guangliang

Chen, Lingen

Chen, Min

Chen, Sheng

Chen, Swe-Kai

Chen, Wei

Chen, Wen

Chen, Yan-chang

Cheng, Shyi-Chyi

Cherpeau, Nicolas

Chia, Nicholas

Chignell, Mark

Chiu, Chih-Chou

Cho, Hyun Cheol

Chon, Tae-Soo

Chramcov, Bronislav

Chuhay, Roman

Cifra, Michal

Ciliberto, Sergio

Cimolin, Veronica

Ciucci, Davide

Ciuonzo, Domenico

Ciuperca, Gabriela

Clote, Peter

Codetta-Raiteri, D.

Coffey, William T.

Cohen-Tanugi, Johann

Colonnese, Stefania

Consolini, G.

Cooch, Evan

Corato, G.

Corda, Christian

Corichi, Alejandro 
Corominas-Murtra, Bernat

Couceiro, Micael

Cross, Daniel J.

Csernak, Gabor

Cucic, Dragoljub A.

Cunliffe, Andrew

Dagger, Ernesto Medina

Dahlsten, Oscar C O

Dalla Torre, Emanuele G.

Darve, Eric

DasGupta, Bhasker

Datsko, Bohdan

Dauwels, J.

Davidson, Aharon

Daw, C. Stuart

De Boer, Jan

de Bragança Pereira, Carlos

Alberto

De Pessemier, Toon

de Poel, Harjo

De Santis, Angelo

de Vladar, Harold P.

De Zárate, José M. Ortiz

Dedeo, Simon

Dedkov, G. V.

Delgado, Ángel

Delsole, Timothy

Demetrius, Lloyd

Deng, F.-G.

Deng, Nan-Jie

Deorowicz, Sebastian

Descombes, G.

Deutsch, David

Devaty, Robert

di Matteo, Tiziana

Di Mauro, Ernesto

Di Vita, A.

Dikmen, O.

Dimitrova, Elena S.

Dionisio, Andreia

Dittrich, Peter

Dochain, Denis
Doltsinis, Nikos

Dominguez-Sal, David

Donner, R. V.

Dorfman, Jeffrey

Dorfman, Konstantine

D'oria, Marco

Dorval, Alan

Downarowicz, Tomasz

Drory, Alon

Drouet, Emmanuel

Drozdz, Stanislaw

Drummond, Neil

Du, Jiulin

Dufault, Renee

Dunk1, Charles F

Dunning-Davies, Jeremy

Duran, Ivan

Durt, Thomas

Dutta, Sourish

Dutt-Mazumder, Aviroop

Earle, Keith

Ebeling, Werner

Ebert-Uphoff, Imme

Eduardo, González-Olivares

Eichler, Michael

Ekström, Magnus

Eliazar, Iddo

Ellahi, R.

Elomaa, Tapio

Elze, Hans-Thomas

Emerson, Joseph

Engelhart, Aaron

Eom, Cheoljun

Erdas, Andrea

Esker, Paul

Esposito, Salvatore

Evans, Denis

Evans, Martin

Evans, Mike

Evans, Tim S.

Faes, Luca

Fajardo, Pablo
Farina, Alfonso

Feidt, Michel

Feistel, Rainer

Feliu, Vicente

Feng, Li

Ferrante, A.

Ferrara, Emilio

Ferri, Cèsar

Ferro, Alfredo

Filanovsky, Igor

Fistola, Romano

Fleming, C.H.

Flyvbjerg, Henrik

Forsythe, K.W.

Fortuna, Luigi

Frénay, Benoît

Fritz, Tobias

Fronsdal, Christian

$\mathrm{Fu}$, Chong

$\mathrm{Fu}$, Huiqing

$\mathrm{Fu}$, John Y

$\mathrm{Fu}$, Zhengyi

Fuchs, Chris

Fuchs, Hans

Funsten, H. O.

Fyfe, Colin

Galca, A.C.

Galhano, Alexandra

Galland, F.

Gama, João

Gao, Michael C.

García-García, Reinaldo

Gardini, Laura

Gaspard, Pierre

Gencaga, Deniz

Geng, Chao-Qiang

Genoverse, Marco

Georgiou, Tryphon T.

Geva, Eitan

Ghani, Nasir

Ghoraani, Behnaz

Gibbs, Adrian 
Giffin, Adom

Giorgi, Franco

Giovanazzi, Stefano

Gkigkitzis, Ioannis

Godang, Romulus

Goh, Kwang-Il

Goldemberg, José

Goldenfeld, Nigel

Goldschmidt, Elizabeth

Goldstein, S. Goldstein

Gompper, Gerhard

Gorban, Alexander

Gough, Michael

Goyal, Philip

Grabowicz, P.a.

Graff, Grzegorz

Graham, D. B.

Graham, Daniel

Grassberger, Peter

Grassia, Filippo

Grassl, Markus

Graves, Daniel

Graziano, Giuseppe

Grechuk, Bogdan

Grendar, Marian

Grimm, Uwe

Gromov, Vasilii A.

Gross, David

Grosskinsky, Stefan

Gruber, Andreas

Gruebele, Martin

Gryder, Berkley E.

Gsiopsis, Abc

Gude, Veera Gnaneswar

Guidotti, Patrick

Guillaume, Jean-Loup

Guivant, Jose

Guo, Sheng

Guojie, Li

Haddadi, Hamed

Hajdu, J.

Hajiaboli, Mohammad Reza
Hamada, Michiaki

Hand, David

Hanel, Rudolf

Hansen, Alex

Hansen, J. S.

Haranas, Ioannis

Harremoes, Peter

Hartkamp, Remco M.

Haruna, Taich

Hautamaki, Ville

He, Yaling

Hébrard, Guillaume

Heinemann, Jack

Hempel, Matthias

Henri, P.

Heremans, Joseph

Herman, Michael

Hernández-Saldaña, H.

Herrera, Roberto Henry

Herwig, Heinz

Hidalgo, Arturo

Ho, Mae-Wan

Hoffmann, Karl Heinz

Hoffmann, Matej

Hofman, T.

Homma, Noriasu

Honrubia-Escribano, A.

Horng, Ming-Huwi

Horrocks, Benjamin R.

Hou, Daqing

Houghton, Conor J.

Hruschka, Daniel J.

Hsiao, Bo

Hsieh, Ker-Chang

Hsieh, Min-Hsiu

Hu, Qiang

$\mathrm{Hu}$, Suxing

Huang, G.H.

Huang, Wei

Hudson, H. S.

Hughes, Keith H.

Hung, F.
Hung, Feiyi

Hutter, Jürg

Hutter, Marcus

Ibañez, Guillermo

Ihle, Thomas

Imre, Emoke

Ince, Robin

Isar, Aurelian

Isidro, Jose

Iyengar, Srinivasan $\mathrm{S}$.

Jacobs, Donald J.

Jagielski, Maciej

Jamil, Mubasher

Jata, Kumar V.

Jha, Basant K.

Jiang, Haijun

Jiang, P.X.

Joergenssen, Sven Erik

Jona-Lasinio, Giovanni

Jones, Christopher K. R. T.

Jones, Daniel

Joshi, C.

Jou, David

Jurman, Giuseppe

Kachenoura, Amar

Kajdanowicz, Tomasz

Kalogeropoulos, Nikolaos

Kaltenbrunner, Andreas

Kampermann, Hermann

Kaniadakis, G

Kaniadakis, Giorgio

Kao, Yucheng

Kaplan, Ehud

Karaiskos, Nikos

Karasuyama, Masayuki

Karathanassis, Ioannis K.

Kaschesky, Michael

Katsaprakakis, Dimitris Al.

Katsoulakis, Markos

Katushkina, Olga

Kaul, Brian

Kauranne, Tuomo 
Kavouras, Marinos

Kel, Oksana Viktorovna

Keller, A.

Keller, K.

Kenett, Dror

Kershenbaum, Arik

Kestler, Hans Armin

Khalil, Khalil

Khan, Asifullah

Kim, Hyang Mi

Kim, Kisik

Kim, Min Soo

Kinney, Justin

Kiryu, Hisanori

Kittichokechai, Kittipong

Kjelstrup, Signe

Kliesch, Martin

Knuth, Kevin H

Kohler, Jurgen

Koomen, E.

Koroli, V. I.

Koslowsky, David

Kosmidou, Vasiliki E.

Kostal, Lubomir

Kostic, Milivoje

Koukouvinos, Christos

Kowalik-Urbaniak, Ilona

Kreutz, Christoph

Kuai, Le

Kukar, Matjaz

Kumar, Mrinal

Kumar, Parmod

Kvasnica, Michal

Kyprianidis, I.M.

Labys, Walter

Laederach, Alain

Laio, Alessandro

Lakshminarayanan, Balaji

Lambiotte, Renaud

Landini, Fabio

Lane, Richard

Laner, David
Larson, Jonas

Larue, James P.

Laso, Manuel

Lasser, Caroline

Lau, Francis

Laurent, Monique

Laurie, J.

Leck, C.

Leclair, Andre

Leduc, Matt

Lee, Cheng-I

Lee, Jejung

Lee, Rob

Leeds, Stephen

Lehar, Alfred

Lei, Yaguo

Leitgeb, Erich

Lenzi, Ervin Kaminski

Lesne, Annick

Leubner, Manfred

Levinson, David Matthew

Levy-Varon, Jennifer

Li, Changpin

Li, Chengqing

Li, Gang

Li, Jie

Li, Jun-bao

Li, S.J.

Li, Wei

Lian, Shuang-Shii

Liang, Hualou

Liang, Xingang

Liberal, Fidel

Lilieholm, Rob

Lin, Chih-jer

Lin, Chun-Ming

Lin, Su-Jien

Lin, Tsung-Chih

Lineweaver, Charles

Linsky, Jeffrey

Lion, A.

Lissia, M.
Litak, Grzegorz

Liu, C. T.

Liu, Chen-hao

Liu, Meizhu

Liu, Xinge

Liu, Xingwen

Liu, Yuan

Liu, Zhengjun

Livadiotis, George

Lizier, Joseph

Lizier, Joseph T.

Lloyd, Seth

Lo Franco, Rosario

Lock, Eric F.

Lorenz, Ralph

Lori, Nicolás F.

Lorite, I.

Louis-Martinez, Domingo J.

Lowe, Will

Lu, Jianfeng

Lu, Yang-Ming

Lucia, Umberto

Luo, Albert C. J.

Luo, Shunlong

Lupo, Cosmo

Luque, Bartolome

Lushi, Enkeleida

Luukka, Pasi

Lv, Jinhu

M. Mahmoud, Gamal

M. Puerta, José

Ma, Hongan

Ma, Zhan

Maas, Jan

Macau, Elbert

Macauley, Matt

Madden, L.

Maeda, Kenichi

Magin, Richard

Mahdian, Babak

Main, P.

Mainardi, Francesco 
Maitra, Neepa

Man, Zhihong

Mancini, Stefano

Mani, A.

Mannheim, Philip

Manzano, D.

Mareschal, Michel

Margarita, Kokla

Mari, Romain

Marinakis, Yannis

Marinazzo, Daniele

Maritan, Amos

Marques, Glauber Tadaiesky

Marques, Ion

Marre, Olivier

Marsili, Matteo

Martin, Ryan

Martin-Martinez, Eduardo

Martyushev, Leonid

Maruyama, Koji

Maslov, Anatoly

Matala-aho, Tapani

Mathias, Gerald

Mathis, Wolfgang

Matsuno, Koichiro

Matsuzoe, Hiroki

Matthews, Miccal Timothy

Mcateer, James

Mcleod, Kenneth

Meister, Bernhard K.

Melchior, Pierre

Meloni, Sandro

Merker, Jochen

Mertsching, Baerbel

Meyn, Jan-Peter

Mezghani, Neila

Miccichè, Salvatore

Mignotte, Max

Miller, Thomas

Milton, Kimball

Miritello, Giovanna

Mistry, Karan H.
Mnatsakanov, Robert

Mocenni, Chiara

Mohsennia, Mohsen

Molenaar, Peter

Molteni, Carla

Monard, Gerald

Mondal, Debashis

Micharet, C.A.M.

Monson, Peter

Montangero, Simone

Montero Sousa, Juan Aurelio

Mookiah, Muthu Rama

Krishnan

Mora, Thierry

Morabito, Francesco Carlo

Morales-Hernánde, M.

Morán, Antonio

Moreau, Michel

Morio, Jérome

Moroni, Saverio

Mosekilde, Erik

Mosier, A.R.

Motsa, Sandile

Mueller, Ingo

Muga, Juan Gonzalo

Mulder, Joris

Mundy, Chris

Muñoz, José J.

Munro, Bill

Mureika, J.R.

Nakagawa, Kenichi

Naudts, Jan

Navarro, Noemi

Néda, Zoltán

Neirotti, Juan

Neupane, Ishwaree

Newton, Nigel

$\mathrm{Ng}$, Soon Xin

Nicolaou, Nicoletta

Nicolis, G.

Niemiec, Marcin

Nien, H.H.
Nieto, Juan J.

Nightingale, James

Nitschke, Wolfgang

Nivanen, L.

Niven, Robert

Nobre, Fernando

Noguchi, Hiroshi

Noh, Jae Dong

Noh, Yung-Kyun

Nojiri, Shin'ichi

Nomura, Shusaku

Nori, Franco

Norton, John D.

Nosonovsky, Michael

Nouadje, Brigitte Astrid Medjo

Obradors, X.

Okatan, Murat

Olchanyi, Maxim

Olivares, F.

Oliveira, Paulo Moura

Olivetti, Emanuele

Omi, Takahiro

Osiejuk, Tomasz S.

Oulhadj, Hamouche

Owari, Maskai

Oztop, Hakan F.

Pagonabarraga Mora, Ignacio

Pahlavani, Mohammad Reza

Paillard, Didier

Paja, Wieslaw

Panoff, Jean- Michel

Papana, A.

Papapetrou, M.

Pappas, Nikolaos

Paris, Jean

Paris, Jeff

Park, Chul Gyu

Park, Ju H.

Parra, Ruben D.

Parry, Matthew

Pascale, Salvatore

Patel, Sandeep 
Peel, Leto

Pennini, F.

Pérez, José A. Moreno

Perez, Liliana

Pérez-Madrid, A.

Perrier, Edith

Pessa, Eliano

Pettigrew, Richard

Piantanida, Pablo

Pierro, Gaetano

Pietsch, Wolfgang

Pillow, Jonathan W.

Pinheiro, Aluisio de Souza

Pinto, Carla

Pipa, Gordon

Pires, E.J. S.

Pistone, Giovanni

Pitard, E.

Pizzolato, N

Planat, Michel

Plastino, A.

Plastino, Angel R.

Pluchino, Alessandro

Pogromsky, A.y.

Poisot, Timothée

Pollack, Gerald H.

Suganthan, P.N.

Pop, Ioan

Portillo, José

Poulsen, Jens

Pourghasemi, Hamid Reza

Prior, Javier

Prokopenko, Mikhail

Pugliese, Daniela

Pumain, Denise

Qadrdan, M

Qi, Donglian

$\mathrm{Qu}$, Xiaodong

Quarati, P.

Queirós, Sílvio M. Duarte

Quintian Pardo, Héctor

Rajamani, Sudha
Rajput, B. S.

Rakthanmanon, Thanawin

Rallo, Robert

Ranjbar, N. A.

Rau, A. Ravi P.

Rauh, Johannes

Ray, Santanu

Read, Daniel

Rech, Paulo

Redelico, Francisco

Rees, Bradley S.

Reeves, Howard

Regal, Cindy

Reidys, Christian M.

Reinard, A. A.

Ren, Mifeng

Restuccia, Liliana

Reuter, Martin

Rhouma, Rhouma

Ribas, Imma

Ribeiro, Haroldo Valentin

Richard, John P.

Richert, Clemens

Ridolfi, Elena

Rigby, James Robert

Rigosa, J.

Riguzzi, Fabrizio

Riveiro, Maria

Rodríguez, Rosalío F

Roff, Greg

Rogatko, Andre

Romano, Vittorio

Rondoni, Lamberto

Rossa, Fabio Della

Rostami, Mohammad

Rottler, Jörg

Rovelli, Carlo

Rowlands, Andrew S.

Roy, Daniel

Roy, Debjani

Rubi, J. Miguel

Rubio, Ramón G.
Ruddell, Ben

Rudoy, Yu. G.

Ruiz, Francisco J. R.

Ruiz, Rubén

Russell, Stephen

Ryu, Keun Ho

Saakes, Michel

Saco, P.m.

Sadiki, Amsini

Sagawa, Takahiro

Sahin, Ahmet

Salanne, Mathieu

Saleh, Ahmad Abou

Sales, Antonio

Sales, Bruno B.

Santiago, Mazuelas

Santodonato, Lou

Santos Corchero, Emilio

Saridakis, Emmanuel N.

Sasa, Shin-ichi

Sato, Aki-Hiro

Sawada, Yasuji

Sawicki, Ignacy

Scala, Antonio

Schaefer, Rafael

Schehr, Gregory

Schilling, Marcel

Schmelzer, Jürn W.p.

Schmitt, Pamela

Schneeloch, James

Schneider, Lorenz

Sciubba, Enrico

Seabright, Paul

Semiat, Raphael

Senkov, Oleg N.

Séralini, Gilles-Eric

Sergeyev, Yaroslav

Sessa, Salvatore

Seth, Sohan

Sfetsos, Athanasios

Shamir, Lior

Sharov, Alexei A. 
Sharpee, Tatyana

Shaw, Christopher

Shen, Shengping

Shikano, Yutaka

Shin, Soo-Young

Shiplyuk, Alexander

Shpyrko, Serge

Shu, Longcang

Siegel, David

Siegle, Malte

Silva, Pedro

Sima, Chao

Singh, Abhinav

Singh, Shailesh Kumar

Singh, V. P.

Singleton, Douglas

Sinitsyn, Nikolai

Sivasundaram, Seenith

Skokos, Charalampos

Skowron, Andrzej

Slater, Paul B

Sleigh, Jamie

Smítal, J.

Smith, Eric

Snoke, D.W.

Sodano, Pasquale

Sohl, Terry

Sommerlade, Linda

Song, Chaoming

Song, Joon Jin

Song, Lixin

Sorrentino, Francesco

Spain, Madrid

Spallucci, Euro

Srinivasa, Arun

Stamatis, Anastassios G.

Stamovlasis, Dimitrios

Stanek, Wojciech

Stanley, Gene

Stanton, Samuel C.

Starikov, E. B.

Stauffer, Dietrich
Steele, Russell

Stehlík, Milan

Steinhaeuser, Karsten

Stentoft, Lars

Stolz, Gabriel

Stover, Rick

Stoy, Paul

Stoyanov, Jordan

Succi, Sauro

Sullivan, Tim

Sun, Jitao

Susemihl, Alex

Suweis, Samir

Svozil, Karl

Syam, Muhammed

Tadic, Bosiljka

Tadić, Bosiljka

Takada, Akira

Takahashi, Tetsuya

Takeuchi, Akira

Tapiero, Oren J.

Tchanche, B.f.

Tekbas, Onderhaluk

Teng, Jen-Hao

Teodoro, George

Theil, Florian

Theodorou, Evangelos A.

Thims, Libb

Thissen, R.

Thoens, Sebastian

Thurner, Stefan

Tian, Fuyang

Tildesley, Dominic

Torres, Miguel

Trell, Erik

Trigeassou, Jean-Claude

Trivellato, Barbara

Tsao, L.C.

Tsekoura, Konstantinos

Tsuchiya, Taku

Tuckerman, Mark

Tumminello, Michele
Turgut, Sadi

Tuszynski, Jack

Typel, S.

Ubøe, Jan

Uffink, Jos

Uohashi, Keiko

Urmossy, K.

Ust, Yasin

Vaccaro, Gianfranco

Vacha, Lukas

Valek, P. W.

Valero, J.

Van Lelyvelda, Iman

Van Willigenburg, L. G.

Ván, Péter

Vanden-Eijden, Eric

Vasyliūnas, V. M.

Velasco, M. Pilar

Velasco, Sergio

Velazques, Luisberis

Venegas-Andraca, Salvador

Verdoolaege, Geert

Versteeg, Greg

Vestroni, Fabrizio

Vicente, Raul

Vidyasagar, M.

Vinck, Martin

Vitiello, Giuseppe

Vitos, Levente

Vlad, Cristina

Volos, Ch.K.

VonSpakovsky, Michael

Vourdas, A.

Vrabec, Jadran

Wada, Tatsuaki

Walk, Nathan

Wallman, Joel

Wang, Anzhong

Wang, Can

Wang, Chuan

Wang, Haimin

Wang, Han 
Wang, Hao

Wang, Jianhui

Wang, Jingfeng

Wang, Lixing

Wang, Q. Alexandre

Wang, Xing-yuan

Wang, Xufei

Wang, Yi-Chi

Wang, Yongtao

Warren, Patrick

Waters, Nigel

Watkins, Chris

Weaver, Iain

Weber, Gerhard Wilhelm

Wei, Junjie

Weng, H.C.

Wentzcovitch, Renata

Whitmore, A.P.

Wiegelmann, Thomas

Wiese, Moritz

Wiesner, Karoline

Wilde, Mark M.

Will, Sebastian

Wilson, Alan

Winsberg, Eric

Witt, Karl J.

Wolpert, David
Wolsztynski, Eric

Wouagfack, Paiguy Armand

Ngouateu

Wu, Xing

Xiao, Junhao

Xie, Lei

$\mathrm{Xu}, \mathrm{Ze}-$ Shui

$\mathrm{Xu}$, Zhengquan

Yablonsky, Gregory

Yamano, Takuya

Yamazaki, Yasunori

Yan, Weimon

Yang, Shaowu

Yasar, Murat

Yeh, An-Chou

Yen, C.W.

Yeoh, William

Yin, Peng-Yeng

$\mathrm{Yu}$, Haijun

Yu, Mei

$\mathrm{Yu}$, Yongguang

Zabaras, Nicholas

Zaidi, Abdellatif

Zamiyatin, Alexander

Zanardi, Paolo

Zanin, Massimiliano

Zeilinger, Anton
Zeng, Nianyin

Zerbetto, Francesco

Zhang, Baicheng

Zhang, Lin

Zhang, Qingling

zhang, Wei

Zhang, Weiping

Zhang, Yong

Zhang, Yvette Yu

Zhang, Zhi

Zhao, Ming

Zheng, Kai

Zhou, Shengxi

Zhu, Fangqiang

Zhu, Pinwen

Zhu, Qiji Jim

Zhuang, Jun

Zhusubaliyev, Zhanybai T.

Ziv-Ukelson, Michal

Zjavka, L.

Zmeskal, Oldrich

Zografos, K.

Zrelly, Yasamine

Zulvia, Ferani E.

Zupanovic, Pasko

Zurek, Sebastian

Zyczkowski, Karol

(C) 2014 by the authors; licensee MDPI, Basel, Switzerland. This article is an open access article distributed under the terms and conditions of the Creative Commons Attribution license (http://creativecommons.org/licenses/by/3.0/). 